\title{
Localized non-diffusive asymptotic patterns for nonlinear parabolic equations with gradient absorption
}

\author{
Philippe Laurençot¹ and Juan Luis Vázquez?2
}

Dedicated to Pavol Brunovský

\begin{abstract}
We study the large-time behaviour of the solutions $u$ of the evolution equation involving nonlinear diffusion and gradient absorption

$$
\partial_{t} u-\Delta_{p} u+|\nabla u|^{q}=0 .
$$

We consider the problem posed for $x \in \mathbb{R}^{N}$ and $t>0$ with non-negative and compactly supported initial data. We take the exponent $p>2$ which corresponds to slow $p$-Laplacian diffusion, and the exponent $q$ in the superlinear range $1<q<p-1$. In this range the influence of the Hamilton-Jacobi term $|\nabla u|^{q}$ is determinant, and gives rise to the phenomenon of localization. The large time behaviour is described in terms of a suitable self-similar solution that solves a Hamilton-Jacobi equation. The shape of the corresponding spatial pattern is rather conical instead of bell-shaped or parabolic.
\end{abstract}

\section{Introduction}

Researchers have been interested for decades in the long time description of evolution processes where diffusion is combined with other effects, notably reaction, absorption and/or convection. The equations under study are evolution equations of parabolic type, mostly nonlinear and possibly degenerate parabolic. A general form of such equations is

$$
\partial_{t} u=\sum_{i=1}^{N} \partial_{x_{i}} A_{i}(t, x, u, \nabla u)+B(t, x, u, \nabla u),
$$

where the $A_{i}$ and $B$ are nonlinear functions with suitable structure conditions as in [30], and $\nabla u$ denotes the spatial gradient of $u$. Under such a generality one cannot be very specific about the concrete asymptotic behaviour of the solutions as $t \rightarrow \infty$. On the contrary, when one concentrates into specific forms for $A_{i}$ and $B$, and also imposes specific boundary conditions, or works in the whole space for concrete classes of initial data, then much is known about the long time behaviour of the solutions, and the different types of possible behaviours have been carefully classified, or are in the process of being classified.

\footnotetext{
${ }^{1}$ Institut de Mathématiques de Toulouse, CNRS UMR 5219, Université Paul Sabatier (Toulouse III), 118 route de Narbonne, F-31062 Toulouse Cedex 9, France. E-mail: laurenco@mip.ups-tlse.fr

${ }^{2}$ Departamento de Matemáticas, Universidad Autónoma de Madrid, Campus de Cantoblanco, E-28049 Madrid, Spain. E-mail: juanluis.vazquez@uam.es
} 
Following this spirit, we will concentrate here on equations where the right-hand side of the equation (11) combines nonlinear diffusion of the $p$-Laplacian type with nonlinear absorption of Hamilton-Jacobi type. We pose the evolution on $\mathbb{R}^{N}, N \geq 1$, as spatial domain, and take as initial condition a non-negative and integrable function $u_{0}$ decaying sufficiently rapidly to zero as $|x| \rightarrow \infty$ (we may assume $u_{0}$ to be compactly supported and bounded to make the analysis simpler). Under these assumptions, we want to classify the different types of long type behaviours that may arise from the interaction between the two physical effects at work. As a new contribution to the topic, we describe in detail a situation in which the evolution leads to localized patterns with a precise power-like decay in time and conical spatial shape.

\subsection{The case of linear diffusion}

Before we enter into that study and in order to motivate the issue, it will be useful to briefly recall the main results in the case of standard linear diffusion. The large time behaviour of nonnegative and integrable solutions to the diffusive Hamilton-Jacobi equation (also called viscous Hamilton-Jacobi equation)

$$
\partial_{t} u-\Delta u+|\nabla u|^{q}=0 \quad \text { in } \quad Q:=(0, \infty) \times \mathbb{R}^{N},
$$

has been studied recently by several authors. It turns out that such behaviour is not unique and strongly depends on the value of the parameter $q \in(0, \infty)$. More precisely, always for initial data decaying sufficiently rapidly to zero as $|x| \rightarrow \infty$, the analysis reveals the existence of two critical exponents $q_{1}:=1$ and $q_{*}:=(N+2) /(N+1)$, and three different types of large time behaviour corresponding to the parameter intervals $q \in\left(0, q_{1}\right), q \in\left(q_{1}, q_{*}\right)$, and $q \in\left(q_{*}, \infty\right)$, respectively. To this we must add the study of the critical cases $q=1$ and $q=q_{*}$.

To be more precise, in the upper range $q>q_{*}$, the large time dynamics is governed by the sole diffusion term and $u$ behaves as a multiple of the fundamental solution to the linear heat equation [11, 17]. After convenient renormalization we thus see the typical bell-shaped Gaussian profile, in the sense that

$$
t^{N / 2} u(t, x) \rightarrow F(y)=c e^{-y^{2} / 2}
$$

with $y=x / t^{1 / 2}$, just as the same form as in the case with no absorption. This is a case of what is called in [34] asymptotic simplification, and it also happens for the semilinear equation $\partial_{t} u-\Delta u+u^{r}=0$ in $Q$ when $r>r_{*}:=(N+2) / N$.

In the intermediate range $q \in\left(1, q_{*}\right)$, the large time behaviour results from the combined effects of the diffusion and absorption terms and is fully described by the self-similar Very Singular Solution to equation (21), cf. [11]. The pattern that we see after the corresponding renormalization, $F_{q}(y)$, is a modification of the Gaussian profile corresponding to the Very Singular Solution and changes with $q$.

As usual in dynamical studies, the large time behaviour for the critical exponents $q_{1}$ and $q_{*}$ is peculiar. For $q=q_{*}$ it is still given by a multiple of the fundamental solution to the linear heat equation but with extra logarithmic factors resulting from the fact that the dynamics already feels the effects of the absorption term [22]. This is usually referred to as resonance.

On the other hand, when $q$ equals $q_{1}=1$ then (2) is still a nonlinear equation but with the same homogeneity as a linear equation, and the large time behaviour does not seem to be thoroughly 
understood. Nevertheless, there are several possible temporal decay rates for $u$ depending on the initial condition [13, 14]. This is an important issue that needs further investigation.

Finally, when the parameter $q$ is below $q_{1}$ the nonlinear absorption term becomes dominant and diffusion plays a secondary role for large times. The typical phenomenon of the lower range is finite time extinction, which takes place for $q \in\left(0, q_{1}\right)$ [12, 23]. Summarizing, the nonlinear absorption term only prevails for large times if $q \in\left(0, q_{1}\right)$. But the exponent $q_{1}=1$ is actually somehow "doubly" critical: indeed, not only does the nonlinearity rule the dynamics but it is also no longer locally Lipschitz continuous for $q \in\left(0, q_{1}\right)$, thus giving rise to singular phenomena such as finite time extinction.

\subsection{Nonlinear diffusion. Localized non-diffusive patterns}

In an attempt to elucidate the true role of the absorption term when it governs the dynamics, we devote this paper to investigate in detail the large time behaviour of non-negative solutions $u$ to the related Cauchy problem

$$
\begin{aligned}
\partial_{t} u-\Delta_{p} u+|\nabla u|^{q} & =0, \quad(t, x) \in Q, \\
u(0) & =u_{0} \geq 0, \quad x \in \mathbb{R}^{N},
\end{aligned}
$$

where the linear diffusion operator $\Delta u$ is replaced by the $p$-Laplacian operator

$$
\Delta_{p} u:=\operatorname{div}\left(|\nabla u|^{p-2} \nabla u\right) .
$$

When $p>2$, (3) is a quasilinear degenerate parabolic equation which reduces to the semilinear equation (2) for $p=2$, and has been studied recently: the existence and properties of self-similar solutions are investigated in [25, 33], while qualitative properties of non-negative and integrable solutions are obtained in [1, 10, 37]. In particular, two critical exponents have been recently identified for (3) [1, 10], namely

$$
q_{1}:=p-1 \quad \text { and } \quad q_{*}:=p-\frac{N}{N+1} .
$$

As for $p=2$, the nonlinear absorption term governs the large time dynamics for $q \in\left(0, q_{1}\right)$ but we now have $q_{1}>1$ since $p>2$. However, the singular phenomena like extinction in finite time are only expected to happen in a still lower subregion $q \in(0,1)$.

Consequently, in the new intermediate range $q \in\left(1, q_{1}\right)$ that we want to explore here the nonlinearity is locally Lipschitz continuous and no singular phenomenon (such as extinction) can occur. Still, the absorption term strongly affects the evolution for $q \in\left(1, q_{1}\right)$. Two results are established in [10] in that parameter interval for compactly supported initial data: there are positive constants $C>0$ and $R>0$ (possibly depending on the initial data) such that

$$
\mathcal{P}_{u}(t) \subset B(0, R) \text { and }\|u(t)\|_{\infty} \leq C t^{-1 /(q-1)}
$$

for all $t \geq 0$ [here and below we use the notation $\mathcal{P}_{u}(t)=\left\{x \in \mathbb{R}^{N}: u(t, x)>0\right\}$ for the positivity set of the function $u$ at time $t$ and we use the abbreviated notation $u(t)$ instead of $u(t, \cdot)$ and so on, whenever there is no fear of confusion]. 
These properties are reminiscent of those enjoyed by the viscosity solutions to the Hamilton-Jacobi equation

$$
\partial_{t} h+|\nabla h|^{q}=0 \quad \text { in } \quad Q
$$

with compactly supported initial data. Indeed, denoting by $h$ the viscosity solution to equation (6) with initial condition $h(0)=u(0)$, we have for each $t \geq 0$ (cf. [7])

$$
\sup _{t \geq 0}\left\{t^{1 /(q-1)}\|h(t)\|_{\infty}\right\}<\infty, \quad \text { and } \quad \mathcal{P}_{h}(t)=\mathcal{P}_{h}(0)
$$

This is in sharp contrast with the behaviour of the solution $w$ to the pure $p$-Laplacian equation $\partial_{t} w-\Delta_{p} w=0$ in $(0, \infty) \times \mathbb{R}^{N}$ with the same initial condition $w(0)=u(0)$. Indeed, the $L^{\infty}$-norm of $w(t)$ only decays at the much slower rate

$$
\|w(t)\|_{\infty}=O\left(t^{-N /(N(p-2)+p)}\right)
$$

and the positivity set $\mathcal{P}_{w}(t)=\left\{x \in \mathbb{R}^{N}: w(t, x)>0\right\}$ of $w(t)$ expands and as $t \rightarrow \infty$ it fills the whole space $\mathbb{R}^{N}$. The absorption term $|\nabla u|^{q}$ in (3) thus prevents this expansion if $q \in\left(1, q_{1}\right)$ and the positivity set of $u(t)$ remains localized in a fixed ball of $\mathbb{R}^{N}$ for all times.

The property of localization is already known to be satisfied by compactly supported non-negative solutions to second-order degenerate parabolic equations with a sufficiently strong absorption involving the solution only as, for instance, $\partial_{t} w-\Delta_{p} w+w^{r}=0$ in $Q$ when $r \in(1, p-1)$, cf. [21, 27, 37]. It had apparently remained unnoticed for second-order degenerate parabolic equations with an absorption term depending solely on the gradient.

According to the previous discussion, the qualitative results obtained so far on compactly supported non-negative solutions to (3) show evidence of the domination of the nonlinear absorption term for large times. The purpose of this paper is to go one step further in that direction and show that $u(t)$ behaves like a self-similar solution to (6) as $t \rightarrow \infty$.

For comparison, it is interesting to notice that this localized asymptotics is quite different in shape from the behaviour of the diffusion-absorption equation $\partial_{t} w-\Delta w^{m}+w^{r}=0$ in $Q$ that offers a strong similarity in other respects with our present problem when $1<r<m$. The corresponding localized behaviour has been studied in [15, 16, 19] and leads to a mesa-like pattern. In this paper we show that a conical pattern $V_{\infty}$ is gradually formed, precisely given by formula (15), and is actually a viscosity solution to the stationary equation

$$
|\nabla v|^{q}-v=0 \text { in } \mathbb{R}^{N}
$$

It is associated to an exact self-similar viscosity solution of the Hamilton-Jacobi equation (6).

As a final contribution, we investigate conditions under which the support does not move at all, so-called infinite waiting time, so that what happens for the whole time span is a process of internal reorganization. We refer to [4] and [36] for waiting times in porous medium flows. In those cases the waiting time is always finite. An infinite waiting time is described in [18, 19] for the equation $\partial_{t} w-\Delta w^{m}+w^{r}=0$ in $Q$ when $1<r<m$. Properties of localization and waiting times for more general equations of the form (11) are considered for instance in [2, 3, 21]. 


\section{Preliminaries and main results}

Before stating our results, let us first specify our assumptions and recall the properties of solutions to (3), (4) with non-negative and compactly supported initial data established in [10]. We assume that

$$
p>2 \text { and } q \in(1, p-1),
$$

and that the initial condition $u_{0}$ enjoys the following properties:

$$
u_{0} \in W^{1, \infty}\left(\mathbb{R}^{N}\right), \quad u_{0} \geq 0, \quad \mathcal{P}_{0}:=\left\{x \in \mathbb{R}^{N}: u_{0}(x)>0\right\} \subset B\left(0, R_{0}\right), u_{0} \not \equiv 0,
$$

for some $R_{0}>0$. Let us also introduce an exponent that will play a role in what follows: $\xi:=$ $1 /(q(N+1)-N)>0$. It goes from $\xi=1$ for $q=1$ to $\xi=1 /(N(p-2)+p-1)$ when $q=p-1$.

Proposition 1 Under the above assumptions, the Cauchy problem (3), (41) has a unique nonnegative viscosity solution

$$
u \in \mathcal{B C}\left([0, \infty) \times \mathbb{R}^{N}\right) \cap L^{\infty}\left(0, \infty ; W^{1, \infty}\left(\mathbb{R}^{N}\right)\right)
$$

which satisfies:

(i) There is $R_{\infty}>0$ such that

$$
\operatorname{supp}(u(t)) \subset B\left(0, R_{\infty}\right) \text { for all } t \geq 0 .
$$

(ii) There are positive constants $C_{0}$ and $C_{1}$ such that

$$
\begin{gathered}
\left\{\begin{array}{l}
\|u(t)\|_{\infty} \leq C_{0}\|u(s)\|_{1}^{q \xi}(t-s)^{-N \xi}, \\
\|\nabla u(t)\|_{\infty} \leq C_{0}\|u(s)\|_{1}^{\xi}(t-s)^{-(N+1) \xi},
\end{array}\right. \\
\|u(t)\|_{1} \leq\left\|u_{0}\right\|_{1}, \quad\|u(t)\|_{\infty} \leq\left\|u_{0}\right\|_{\infty}, \quad\|\nabla u(t)\|_{\infty} \leq\left\|\nabla u_{0}\right\|_{\infty}, \\
t^{1 /(q-1)}\left(\|u(t)\|_{1}+\|u(t)\|_{\infty}+\|\nabla u(t)\|_{\infty}\right) \leq C_{1}
\end{gathered}
$$

for all $t>0$ and $s \in[0, T)$.

Here and below $\mathcal{B C}\left([0, \infty) \times \mathbb{R}^{N}\right)$ denotes the space of bounded and continuous functions on $[0, \infty) \times \mathbb{R}^{N}$ and $\|\cdot\|_{r}$ denotes the $L^{r}\left(\mathbb{R}^{N}\right)$-norm for $r \in[1, \infty]$.

Assertions (10), (11), and (12) are proved in [10, Theorem 1.5 (i)] and [10, Proposition 1.3], respectively, while (13) is established in [10, Corollary 1.6 (i)] for the $L^{1}$-norm. The estimates (13) for $\|u(t)\|_{\infty}$ and $\|\nabla u(t)\|_{\infty}$ follow then from that for $\|u(t)\|_{1}$ by (11) with $s=t / 2$. 

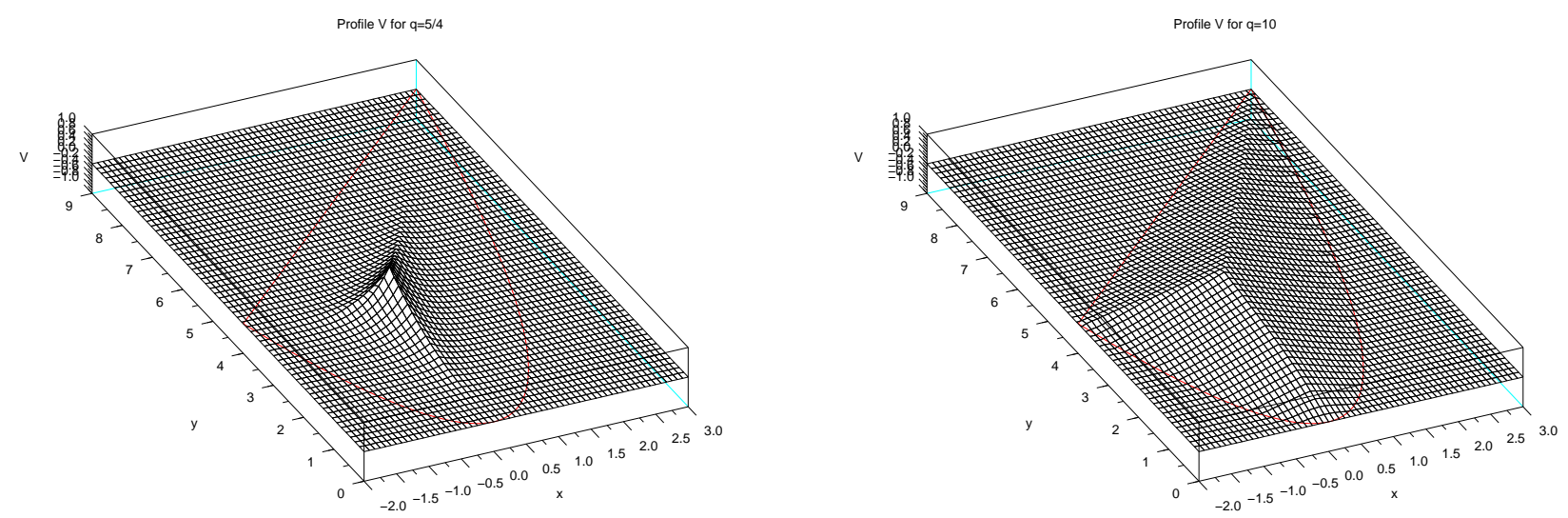

Figure 1: Profile $V_{\infty}$ for $q=5 / 4$ (left) and $q=10$ (right)

\subsection{Statement of the main asymptotic results}

As indicated above we call $\mathcal{P}_{u}(t)$ the positivity set of the solution $u$ at time $t \geq 0$, that we abbreviate as $\mathcal{P}(t)$. Since $u(t)$ is compactly supported and continuous for each $t \geq 0$ by Proposition 1, $\mathcal{P}(t)$ is a bounded open subset of $\mathbb{R}^{N}$ for all $t \geq 0$. Much more can actually be said on the family $\{\mathcal{P}(t)\}_{t \geq 0}$ and it will allow us to identify the large time behaviour of $u$.

Theorem 2 The mapping $t \longmapsto \mathcal{P}(t)$ gives a non-decreasing family of bounded open subsets of $\mathbb{R}^{N}$ and moreover

$$
\mathcal{P}_{\infty}:=\bigcup_{t \geq 0} \mathcal{P}(t) \quad \text { is a bounded open subset of } \mathbb{R}^{N} .
$$

In addition,

$$
\lim _{t \rightarrow \infty}\left\|t^{1 /(q-1)} u(t)-V_{\infty}\right\|_{\infty}=0
$$

where

$$
V_{\infty}(x):=\frac{q-1}{q^{q /(q-1)}}\left(\inf _{y \in \mathbb{R}^{N} \backslash \mathcal{P}_{\infty}}\{|y-x|\}\right)^{q /(q-1)} \quad \text { for } \quad x \in \mathbb{R}^{N}
$$

We first point out that $U(t, x):=t^{-1 /(q-1)} V_{\infty}(x)$ is a self-similar (viscosity) solution to (6) which vanishes outside $\mathcal{P}_{\infty}$ and is positive in $\mathcal{P}_{\infty}$. Therefore, (14) asserts that the diffusion term no longer matters for large times as claimed previously. Still, it might influence the shape of $\mathcal{P}_{\infty}$ during the time evolution. We will come back to this issue in Theorems 3 and 4 below. Also, the final (rescaled) pattern $V_{\infty}$ has a conical shape as illustrated in Figure 1 for $N=2$ and $\mathcal{P}_{\infty}=\left\{(x, y) \in[-2,3] \times[0,9]: x^{2}<y<x+6\right\}$.

The proof of Theorem 2 is divided in several steps and is performed in the forthcoming Sections 3 and 4. We nevertheless outline its main steps now: we first establish the monotonicity property of the family $\{\mathcal{P}(t)\}_{t \geq 0}$ by comparison arguments, and lower bounds for $u(t, x)$ as well. We next turn to the proof of (14) and introduce the self-similar variables; we keep the space variable $x$ (since we 
expect localized behaviour), and (as usual) introduce logarithmic time

$$
\tau:=\ln (1+(q-1) t) /(q-1)
$$

as well as the new unknown function $v=v(\tau, x)$ defined by

$$
u(t, x)=(1+(q-1) t)^{-1 /(q-1)} v(\tau, x), \quad(t, x) \in[0, \infty) \times \mathbb{R}^{N} .
$$

Usually, $v(\tau)=v(\tau, \cdot)$ is called the renormalized trajectory. Clearly, $v$ solves

$$
\begin{aligned}
\partial_{\tau} v+|\nabla v|^{q}-v & =e^{-(p-1-q) \tau} \Delta_{p} v, \quad(\tau, x) \in Q, \\
v(0) & =u_{0}, \quad x \in \mathbb{R}^{N} .
\end{aligned}
$$

The convergence (14) then reads $\left\|v(\tau)-V_{\infty}\right\|_{\infty} \longrightarrow 0$ as $\tau \rightarrow \infty$ and we are thus left to establish the convergence of $v(\tau)$ as $\tau \rightarrow \infty$. We first notice that, as $q<p-1$, the coefficient in front of the diffusion term vanishes for large times, so that the diffusion term is clearly negligible for large times. To identify the large time behaviour of $v(\tau)$ we use the half-relaxed limits technique [9] in the same fashion as in [31, Theorem 1] and [32, Section 3], and introduce

$$
v_{*}(\tau, x):=\liminf _{(\sigma, y, s) \rightarrow(\tau, x, \infty)} v(\sigma+s, y) \quad \text { and } \quad v^{*}(\tau, x):=\limsup _{(\sigma, y, s) \rightarrow(\tau, x, \infty)} v(\sigma+s, y)
$$

for $(\tau, x) \in Q$. We first prove that $v_{*}$ and $v^{*}$ do not depend on $\tau$ and are viscosity supersolution and subsolution, respectively, to the Hamilton-Jacobi equation $|\nabla z|^{q}-z=0$ in $\mathbb{R}^{N}$. Noticing that $v_{*}$ and $v^{*}$ are positive on the same open subset $\mathcal{P}_{\infty}$ of $\mathbb{R}^{N}$, a comparison argument can be applied to conclude that $v_{*}=v^{*}$, from which the convergence of $v(\tau)$ follows.

\subsection{The question of waiting times}

In Theorem 2 we have identified the first term of the expansion of $u(t)$ as $t \rightarrow \infty$. Still there is an unspecified quantity, namely the set $\mathcal{P}_{\infty}$ on which additional information would be welcome. In particular, is there a class of initial data for which the positivity set does not change during time evolution? The next result gives a positive answer to this question.

Theorem 3 Assume that there exists $\delta>0$ such that

$$
\begin{gathered}
u_{0}(x) \leq a_{0}\left|x-x_{0}\right|^{(p-q) /(p-1-q)} \quad \text { for } x \in B\left(x_{0}, \delta\right) \quad \text { and } \quad x_{0} \in \partial \mathcal{P}_{0}, \\
\left\|u_{0}\right\|_{\infty} \leq a_{0} \delta^{(p-q) /(p-q-1)}
\end{gathered}
$$

where

$$
a_{0}:=\frac{p-1-q}{p-q}\left(N-1+\frac{p-1}{p-1-q}\right)^{-1 /(p-1-q)} .
$$

Then $\mathcal{P}(t)=\mathcal{P}_{0}$ for every $t \geq 0$, and thus $\mathcal{P}_{\infty}=\mathcal{P}_{0}$. 
When a point of the boundary of the positivity set stays as such for a time we say that there is a waiting time at that point. Waiting times are typical in degenerate parabolic equations with slow diffusion, like $\partial_{t} w=\Delta w^{m}$ for $m>1$ or $\partial_{t} w=\Delta_{p} w$ for $p>2$, cf. [36], but in these cases they are finite. In the present case we have exhibited an infinite waiting time at all points of the boundary of the initial support.

For small values of $q$, the behaviour reported in Theorem 3 ceases to be observed as soon as (19) is not fulfilled, showing the optimality of the exponent $(p-q) /(p-1-q)$ for the support not to evolve.

Theorem 4 Assume that $x_{0} \in \partial \mathcal{P}_{0}$ and there is $\delta>0$ such that

$$
u_{0}(x) \geq A\left|x-x_{0}\right|^{(p-q) /(p-1-q)} \quad \text { for } \quad x \in B\left(x_{0}, \delta\right) .
$$

If

$$
q<q_{2}:=\min \left\{p-\frac{2 N}{N+1}, \frac{p}{2}\right\}
$$

there is $A_{q}>0$ depending only on $N, p$, and $q$ such that, if $A \geq A_{q}$,

$$
u\left(t, x_{0}\right)>0 \text { for all } t>0 .
$$

In particular, $x_{0} \in \mathcal{P}(t)$ for each $t>0$.

A similar result is actually also valid for $q \in\left[q_{2}, p-1\right)$ but requires a stronger assumption than (22), see Proposition 11 below.

\section{Time monotonicity of the positivity set}

We first establish the time monotonicity of $\mathcal{P}(t)$.

Proposition 5 For $t_{1} \in[0, \infty)$ and $t_{2} \in\left(t_{1}, \infty\right)$ we have $\mathcal{P}\left(t_{1}\right) \subseteq \mathcal{P}\left(t_{2}\right)$ and

$$
\mathcal{P}_{\infty}:=\bigcup_{t \geq 0} \mathcal{P}(t) \quad \text { is a bounded open subset of } \mathbb{R}^{N} .
$$

The proof relies on the construction of a suitable subsolution which we perform next.

Lemma 6 Fix $R>0$ and put

$$
s_{A}(t):=A(1+t)^{-1 /(q-1)}\left(R^{2}-|x|^{2}\right)^{q /(q-1)}, \quad(t, x) \in[0, \infty) \times B(0, R) .
$$

There exists $A_{R}>0$ such that $s_{A}$ is a (viscosity) subsolution to (3) in $(0, \infty) \times B(0, R)$ for every $A \in\left[0, A_{R}\right]$. 
Proof. Introducing $\sigma(x):=\left(R^{2}-|x|^{2}\right)^{q /(q-1)}$ for $x \in B(0, R)$, we observe that $\sigma$ and $|\nabla \sigma|^{p-2} \nabla \sigma$ both belong to $\mathcal{C}^{1}(B(0, R))$. Therefore, if $(t, x) \in[0, \infty) \times B(0, R)$,

$$
\begin{aligned}
& \frac{(1+t)^{q /(q-1)}}{A \sigma(x)}\left\{\partial_{t} s_{A}(t, x)-\Delta_{p} s_{A}(t, x)+\left|\nabla s_{A}(t, x)\right|^{q}\right\} \\
\leq & -\frac{1}{q-1}+\left(\frac{2 q}{q-1}\right)^{q} A^{q-1}|x|^{q} \\
& +\left(\frac{2 q}{q-1}\right)^{q} \frac{(N+p-2) A^{p-2}}{(1+t)^{(p-1-q) /(q-1)}} \sigma(x)^{(p-1-q) / q}|x|^{p-2} \\
\leq & -\frac{1}{q-1}+\left(\frac{2 q R}{q-1}\right)^{q} A^{q-1} \\
& +\left(\frac{2 q}{q-1}\right)^{q}(N+p-2) A^{p-2} R^{(2(p-1-q) /(q-1))+p-2} \leq 0
\end{aligned}
$$

if $A \in\left[0, A_{R}\right]$ where $A_{R}$ is sufficiently small and depends only on $N, p, q$, and $R$.

Proof of Proposition 5. Fix $t_{1} \geq 0$ and $x_{1} \in \mathcal{P}\left(t_{1}\right)$. Owing to the continuity of $x \mapsto u(t, x)$ there are $\delta>0$ and $R>0$ such that $u\left(t_{1}, x\right) \geq \delta>0$ for $x \in B\left(x_{1}, R\right)$. Choosing $A \in\left(0, A_{R}\right)$ such that $A \leq \delta R^{-2 q /(q-1)}$, we notice that

$$
s_{A}\left(t_{1}, x-x_{1}\right) \leq A R^{2 q /(q-1)} \leq \delta \leq u\left(t_{1}, x\right) \text { for } \quad x \in B\left(x_{1}, R\right),
$$

and

$$
s_{A}\left(t, x-x_{1}\right)=0 \leq u(t, x) \quad \text { for } \quad(t, x) \in\left[t_{1}, \infty\right) \times \partial B\left(x_{1}, R\right),
$$

the parameter $A_{R}$ and the function $s_{A}$ being defined in Lemma 6. By Lemma 6, $(t, x) \longmapsto s_{A}(t, x-$ $\left.x_{1}\right)$ is a subsolution to (3) in $\left[t_{1}, \infty\right) \times B(0, R)$ and we infer from the comparison principle [20, Theorem 8.2] that $s_{A}\left(t, x-x_{1}\right) \leq u(t, x)$ for $(t, x) \in\left[t_{1}, \infty\right) \times B(0, R)$. In particular, $u\left(t, x_{1}\right) \geq$ $s_{A}(t, 0)=A(1+t)^{-1 /(q-1)} R^{2 q /(q-1)}>0$ for $t \geq t_{1}$. Therefore, $x_{1} \in \mathcal{P}(t)$ for every $t \geq t_{1}$, which proves the first assertion of Proposition 5. The set $\mathcal{P}_{\infty}$ is then clearly an open subset of $\mathbb{R}^{N}$ which is bounded by (10).

Another useful consequence of Lemma 6 is a lower bound for $u(t)$ which implies in particular that $\|u(t)\|_{\infty}$ cannot decay to zero at a faster rate than $t^{-1 /(q-1)}$ for large times.

Corollary 7 For each $x \in \mathcal{P}_{\infty}$ there are $T_{x} \geq 0$ and $\varepsilon_{x}>0$ such that

$$
u(t, x) \geq \varepsilon_{x}(1+t)^{-1 /(q-1)} \text { for } t \geq T_{x} .
$$

In particular, there exists $C_{2}>0$ such that

$$
\|u(t)\|_{\infty} \geq C_{2} t^{-1 /(q-1)} \quad \text { for } \quad t \geq 1 .
$$

Proof. Consider $x \in \mathcal{P}_{\infty}$. Then there is $T_{x} \geq 0$ such that $x \in \mathcal{P}(t)$ for $t \geq T_{x}$. The continuity of $y \longmapsto u\left(T_{x}, y\right)$ ensures that there are $\delta>0$ and $R>0$ such that $u\left(T_{x}, y\right) \geq \delta>0$ for $y \in B(x, R)$. Choosing $A \in\left(0, A_{R}\right)$ such that $A \leq \delta R^{-2 q /(q-1)}$ we argue by comparison as in the proof of Proposition 5 to conclude that $s_{A}(t, y-x) \leq u(t, y)$ for $(t, y) \in\left[T_{x}, \infty\right) \times B(x, R)$ (recall that $A_{R}$ and $s_{A}$ are defined in Lemma $\left[\right.$ ). Consequently, $u(t, x) \geq s_{A}(t, 0)=A(1+t)^{-1 /(q-1)} R^{2 q /(q-1)}>0$ for $t \geq T_{x}$, whence (25) with $\varepsilon_{x}=A R^{2 q /(q-1)}$. The lower bound (26) is then a straightforward consequence of (25) with any $x \in \mathcal{P}_{0}$. 


\section{Convergence to self-similarity}

We now investigate the properties and convergence for large times of the function $v$ defined in (16). For $\tau \geq 0$ let $\mathcal{P}_{v}(\tau):=\left\{x \in \mathbb{R}^{N}: v(\tau, x)>0\right\}$ be the positivity set of $v(\tau)$. According to (16), $\mathcal{P}_{v}(\tau)$ is given by

$$
\mathcal{P}_{v}(\tau)=\mathcal{P}_{u}\left(\frac{e^{(q-1) \tau}-1}{q-1}\right) \text { for } \quad \tau \geq 0 .
$$

We next state some bounds for the rescaled function $v$ which are easy consequences of (10), (12), (13), and (25).

Lemma 8 There is $C_{3}>0$ such that

$$
\|v(\tau)\|_{\infty}+\|\nabla v(\tau)\|_{\infty} \leq C_{3} \quad \text { for } \quad \tau \geq 0
$$

In addition, for each $x \in \mathcal{P}_{\infty}$, there are $\tau_{x}$ and $\varepsilon_{x}>0$ such that

$$
v(\tau) \geq \varepsilon_{x}>0 \quad \text { for } \quad \tau \geq \tau_{x}
$$

Finally, $\tau \longmapsto \mathcal{P}_{v}(\tau)$ is a non-decreasing family of bounded open subsets of $\mathbb{R}^{N}$ and

$$
\bigcup_{\tau \geq 0} \mathcal{P}_{v}(\tau)=\mathcal{P}_{\infty}
$$

the limit set $\mathcal{P}_{\infty}$ being defined by (24).

Proof. The estimates (27) readily follow from (12), (13), and (16), while (28) and the properties of $\left\{\mathcal{P}_{v}(\tau)\right\}_{\tau \geq 0}$ are straightforward consequences of Proposition 5 and Corollary 7

We turn to our main task, i. e., the behaviour of $v(\tau)$ as $\tau \rightarrow \infty$, and actually aim at showing the convergence of $v(\tau)$ as $\tau \rightarrow \infty$ to an asymptotic profile. For that purpose we use the half-relaxed limits technique [9] in the same fashion as in [32, Section 3]. For $(\tau, x) \in[0, \infty) \times \mathbb{R}^{N}$ we define

$$
v_{*}(x):=\liminf _{(\sigma, y, s) \rightarrow(\tau, x, \infty)} v(\sigma+s, y) \quad \text { and } \quad v^{*}(x):=\limsup _{(\sigma, y, s) \rightarrow(\tau, x, \infty)} v(\sigma+s, y)
$$

and first note that the right-hand sides of the above definitions indeed do not depend on $\tau \geq 0$. In addition,

$$
0 \leq v_{*}(x) \leq v^{*}(x) \text { for } x \in \mathbb{R}^{N}
$$

by (29), while Lemma 8 and the Rademacher theorem clearly ensure that $v_{*}$ and $v^{*}$ both belong to $W^{1, \infty}\left(\mathbb{R}^{N}\right)$. Finally, by [8, Théorème 4.1] applied to equation (17), $v^{*}$ and $v_{*}$ are viscosity subsolution and supersolution, respectively, to the Hamilton-Jacobi equation

$$
H(z, \nabla z):=|\nabla z|^{q}-z=0 \quad \text { in } \quad \mathbb{R}^{N} .
$$


The next step is to show that $v^{*}$ and $v_{*}$ actually coincide. At this point we emphasize that $v^{*}$ and $v_{*}$ enjoy an additional property, namely,

$$
\left\{\begin{array}{lll}
v^{*}(x)=v_{*}(x)=0 & \text { for } & x \in \mathbb{R}^{N} \backslash \mathcal{P}_{\infty}, \\
v^{*}(x) \geq v_{*}(x)>0 & \text { for } & x \in \mathcal{P}_{\infty}
\end{array}\right.
$$

(a similar situation is encountered in the proof of [31, Theorem 1]). Indeed, if $x \notin \mathcal{P}_{\infty}$, the time monotonicity of $\tau \mapsto \mathcal{P}_{v}(\tau)$ (Lemma 8 ) warrants that $x \notin \mathcal{P}_{v}(\tau)$ for all $\tau \geq 0$. Then, if $s_{n} \rightarrow \infty$, $\sigma_{n} \rightarrow 0$, and $x_{n} \rightarrow x$ are such that $v\left(\sigma_{n}+s_{n}, x_{n}\right) \longrightarrow v^{*}(x)$ as $n \rightarrow \infty$, it follows from (27) that

$$
v\left(\sigma_{n}+s_{n}, x_{n}\right) \leq v\left(\sigma_{n}+s_{n}, x\right)+\left|x-x_{n}\right|\left\|\nabla v\left(\sigma_{n}+s_{n}\right)\right\|_{\infty} \leq C_{3}\left|x-x_{n}\right| .
$$

Passing to the limit and recalling (30) gives the first assertion in (32). Consider next $x \in \mathcal{P}_{\infty}$ and recall that there are $\tau_{x}$ and $\varepsilon_{x}>0$ such that $v(\tau, x) \geq \varepsilon_{x}$ for $\tau \geq \tau_{x}$ by (28). Pick sequences $\left(\sigma_{n}\right)_{n \geq 1},\left(s_{n}\right)_{n \geq 1}$, and $\left(x_{n}\right)_{n \geq 1}$ such that $\sigma_{n} \rightarrow 0, s_{n} \rightarrow \infty, x_{n} \rightarrow x$, and $v\left(\sigma_{n}+s_{n}, x_{n}\right) \longrightarrow v_{*}(x)$ as $n \rightarrow \infty$. For $n$ large enough we have $\sigma_{n}+s_{n} \geq \tau_{x}$ and we infer from Lemma 8 that

$$
v\left(\sigma_{n}+s_{n}, x_{n}\right) \geq v\left(\sigma_{n}+s_{n}, x\right)-C_{3}\left|x-x_{n}\right| \geq \varepsilon_{x}-C_{3}\left|x-x_{n}\right| .
$$

Letting $n \rightarrow \infty$ gives $v_{*}(x) \geq \varepsilon_{x}>0$ and completes the proof of (32).

We next introduce

$$
V_{*}(x):=\frac{q}{q-1} v_{*}(x)^{(q-1) / q} \quad \text { and } \quad V^{*}(x):=\frac{q}{q-1} v^{*}(x)^{(q-1) / q}
$$

for $x \in \mathcal{P}_{\infty}$. Arguing as in [8, Corollaire 2.1] or [6, Proposition II.2.5], it easily follows from the properties of $v^{*}$ and $v_{*}$ that $V^{*}$ and $V_{*}$ are viscosity subsolution and supersolution, respectively, to the eikonal equation

$$
H_{e i}(z):=|\nabla z|-1=0 \quad \text { in } \quad \mathcal{P}_{\infty} .
$$

Now, $H_{e i}$ depends solely on $\nabla z$ and is a convex function of $\nabla z$, so that the assumptions (H1), $(\mathrm{H} 2)$, and $(\mathrm{H} 4)$ in [26] are clearly fulfilled. Furthermore, $H_{e i}(0)=-1<0$ which warrants that the assumption (H3) in 26] is also fulfilled (the function $\varphi$ in [26] being here identically zero). Since $V_{*}=V^{*}=0$ on $\partial \mathcal{P}_{\infty}$, we are in a position to apply [26, Theorem 1] to conclude that $V^{*}(x) \leq V_{*}(x)$ for $x \in \mathcal{P}_{\infty}$. Recalling (32) and (33) we end up with $V^{*}(x)=V_{*}(x)$ for $x \in \mathcal{P}_{\infty}$ and thus $v_{*}(x)=v^{*}(x)$ in $\mathbb{R}^{N}$. Owing to [8, Lemme 4.1] or [6, Lemma V.1.9], the equality $v_{*}=v^{*}$ and (29) provide the convergence of $(v(s))_{s \geq 1}$ towards $v_{*}$ uniformly on every compact subset of $\mathbb{R}^{N}$ as $s \rightarrow \infty$. Since all these functions are compactly supported in the closure of $\mathcal{P}_{\infty}$, we conclude that

$$
\lim _{\tau \rightarrow \infty}\left\|v(\tau)-v_{*}\right\|_{\infty}=0 .
$$

Returning to the original variables $(t, x)$, we obtain

$$
\lim _{t \rightarrow \infty}\left\|(1+(q-1) t)^{1 /(q-1)} u(t)-v_{*}\right\|_{\infty}=0,
$$

whence

$$
\lim _{t \rightarrow \infty}\left\|t^{1 /(q-1)} u(t)-(q-1)^{-1 /(q-1)} v_{*}\right\|_{\infty}=0 .
$$


It remains to identify $v_{*}$, or equivalently $V_{*}$. Since the latter is a viscosity solution to $\left|\nabla V_{*}\right|-1=0$ in $\mathcal{P}_{\infty}$ with $V_{*}=0$ on $\partial \mathcal{P}_{\infty}$ by the previous analysis, we infer from [ $\underline{6}$, Remark II.5.10] that

$$
V_{*}(x)=\inf _{y \in \partial \mathcal{P}_{\infty}}\{|y-x|\}=\inf _{y \in \mathbb{R}^{N} \backslash \mathcal{P}_{\infty}}\{|y-x|\} \quad \text { for } \quad x \in \mathcal{P}_{\infty} .
$$

Consequently, $v_{*}=V_{\infty}$, the latter being defined by (15), and (14) follows from (35).

\section{$5 \quad$ Invariant and moving positivity sets}

We first show that, if $u_{0}$ vanishes sufficiently rapidly near a point $x_{0}$, then $u\left(t, x_{0}\right)=0$ for all $t \geq 0$.

Lemma 9 Assume that there are $x_{0} \in \mathbb{R}^{N}$ and $\delta>0$ such that

$$
\begin{gathered}
u_{0}(x) \leq a_{0}\left|x-x_{0}\right|^{(p-q) /(p-1-q)} \quad \text { for } \quad x \in B\left(x_{0}, \delta\right), \\
\left\|u_{0}\right\|_{\infty} \leq a_{0} \delta^{(p-q) /(p-q-1)}
\end{gathered}
$$

the parameter $a_{0}$ being defined by (21). Then,

$$
u\left(t, x_{0}\right)=0 \text { for } t \geq 0 .
$$

Proof. We adapt an argument from [29, Theorem 8.2] and put $S_{1}(x):=a_{0}\left|x-x_{0}\right|^{(p-q) /(p-1-q)}$ for $x \in \mathbb{R}^{N}$. An easy computation shows that

$$
-\Delta_{p} S_{1}(x)+\left|\nabla S_{1}(x)\right|^{q}=0 \text { for } \quad x \in \mathbb{R}^{N} .
$$

Furthermore, we have $u_{0}(x) \leq S_{1}(x)$ for $x \in B\left(x_{0}, \delta\right)$ by (36) and $u(t, x) \leq\left\|u_{0}\right\|_{\infty} \leq S_{1}(x)$ for $(t, x) \in[0, \infty) \times \partial B\left(x_{0}, \delta\right)$ by (12) and (37). The comparison principle [20, Theorem 8.2] then entails that $u(t, x) \leq S_{1}(x)$ for $(t, x) \in[0, \infty) \times B\left(x_{0}, \delta\right)$. Consequently, $u\left(t, x_{0}\right) \leq S_{1}\left(x_{0}\right)=0$ for $t \geq 0$ and the lemma is proved.

Proof of Theorem [3. We first consider $x_{0} \in \partial \mathcal{P}_{0}$. Owing to (19) and (20) we are in a position to apply Lemma 9 to deduce that $u\left(t, x_{0}\right)=0$ for all $t \geq 0$. We next consider $x_{0}$ lying outside the closure of $\mathcal{P}_{0}$ and $x \in B\left(x_{0}, \delta\right)$. Either $x \notin \mathcal{P}_{0}$ and $u_{0}(x)=0 \leq a_{0}\left|x-x_{0}\right|^{(p-q /(p-1-q)}$. Or $x \in \mathcal{P}_{0}$ and there exists $\vartheta \in(0,1)$ such that $x_{\vartheta}:=\vartheta x+(1-\vartheta) x_{0}$ belongs to $\partial \mathcal{P}_{0}$. Then $\left|x-x_{\vartheta}\right|=(1-\vartheta)\left|x-x_{0}\right| \leq \delta$ and we infer from (19) that $u_{0}(x) \leq a_{0}\left|x-x_{\vartheta}\right|^{(p-q /(p-1-q)} \leq$ $a_{0}\left|x-x_{0}\right|^{(p-q /(p-1-q)}$. The assumptions of Lemma 9 are then fulfilled by $u_{0}$ at $x_{0}$ and we conclude again that $u\left(t, x_{0}\right)=0$ for all $t \geq 0$.

We have thus shown that $\mathcal{P}(t) \subset \mathcal{P}_{0}$ for $t \geq 0$. The opposite inclusion readily follows from the time monotonicity of the positivity set established in Proposition 5 .

We now turn to the proof of Theorem 4 and first establish the following result, which is in the spirit of [5, Proposition 3.2]. 
Lemma 10 Consider $q \in\left(1, q_{2}\right), q_{2}$ being defined in (23). There are positive real numbers $C_{4}$ and $C_{5}$ depending only on $N, p$, and $q$ such that, if $u_{0}$ is an initial condition fulfilling (9) and

$$
M:=\int_{B(0,1)} u_{0}(x) d x \geq C_{5},
$$

then the corresponding solution u to (3), (44) satisfies

$$
u(1,0) \geq C_{4} M^{p \eta} \quad \text { with } \quad \eta:=\frac{1}{N(p-2)+p} .
$$

Proof. We adapt the proof given in [35] for the porous medium equation, the main difference being that the $L^{1}$-norm of $u$ is not constant in our case. In the following, we denote by $C_{i}, i \geq 6$, positive constants depending only on $N, p$, and $q$.

Let us first assume that $\operatorname{supp}\left(u_{0}\right) \subset B(0,1)$, so that $\left\|u_{0}\right\|_{1}=M$. Denoting by $w$ the solution to the $p$-Laplacian equation $\partial_{t} w-\Delta_{p} w=0$ in $Q$ with initial condition $w(0)=u_{0}$, the comparison principle entails that $u(t, x) \leq w(t, x)$ for $(t, x) \in[0, \infty) \times \mathbb{R}^{N}$. By [28, Proposition 2.2] and [10, Proposition 1.3] we have

$$
\begin{aligned}
\|u(t)\|_{\infty} & \leq\|v(t)\|_{\infty} \leq C_{6} M^{p \eta} t^{-N \eta} \\
u(t, x) & =v(t, x)=0 \text { if }|x| \geq 1+C_{7} M^{\eta(p-2)} t^{\eta}, \\
\|\nabla u(t)\|_{\infty} & \leq C M^{2 \eta} t^{-\eta(N+1)},
\end{aligned}
$$

for $t>0$, the constants $C_{6}$ and $C_{7}$ depending only on $N, p$, and $q$. We next use the reflection argument of Aleksandrov as in the proof of [5, Lemma 2.2] to deduce from (3) that

$$
u(t, 0) \geq u(t, x) \text { for } t \geq 0 \text { and }|x| \geq 2 .
$$

We then infer from the above bounds that, if $C_{7} M^{\eta(p-2)}>1$,

$$
\begin{aligned}
& C_{8} u(1,0)\left[\left(1+C_{7} M^{\eta(p-2)}\right)^{N}-2^{N}\right] \\
\geq & \int_{\{|x| \geq 2\}} u(1, x) d x \\
\geq & \|u(1)\|_{1}-\int_{B(0,2)} u(1, x) d x \\
\geq & M-\int_{0}^{1} \int_{\mathbb{R}^{N}}|\nabla u(s, x)|^{q} d x d s-C_{8} 2^{N}\|u(1)\|_{\infty} \\
\geq & M-C_{8} \int_{0}^{1}\left(1+C_{7} M^{\eta(p-2)} s^{\eta}\right)^{N}\|\nabla u(s)\|_{\infty}^{q} d s-C_{9} M^{p \eta} \\
\geq & M-C_{10} M^{N \eta(p-2)} M^{2 \eta q)} \int_{0}^{1} s^{-\eta q(N+1)} d s \\
\geq & M-C_{11}\left(M^{\eta(N(p-2)+2 q)}+M^{p \eta}\right)
\end{aligned}
$$


the assumption $q<p-(2 N /(N+1))$ being used to obtain the last inequality. Consequently, if $C_{7} M^{\eta(p-2)}>1$

$$
2^{N} C_{8}\left[C_{7}^{N} M^{N \eta(p-2)}-1\right] u(1,0) \geq M-C_{11}\left(M^{\eta(N(p-2)+2 q)}+M^{p \eta}\right) .
$$

Since $p \eta \in(0,1)$ and $\eta(N(p-2)+2 q) \in(0,1)$ by (23) we readily conclude that there are $C_{12}$ and $C_{13}$ such that $u(1,0) \geq C_{12} M^{p \eta}$ provided $M \geq C_{13}$.

We next consider an arbitrary initial condition $u_{0}$ fulfilling (9). Then there is $\zeta \in \mathcal{C}_{0}^{\infty}\left(\mathbb{R}^{N}\right)$ such that $0 \leq \zeta \leq 1, \operatorname{supp} \zeta \subset B(0,1)$, and

$$
\int_{B(0,1)} \zeta(x) u_{0}(x)=\frac{M}{2}
$$

Denoting by $\tilde{u}$ the solution to (3) with initial condition $\tilde{u}(0)=\zeta u_{0}$, it follows from the above analysis that $\tilde{u}(1,0) \geq C_{12}(M / 2)^{p \eta}$ if $M \geq 2 C_{13}$. As the comparison principle warrants that $u(1,0) \geq \tilde{u}(1,0)$, the expected result follows with $C_{5}=2 C_{13}$ and $C_{4}=C_{12} 2^{-p \eta}$.

Proof of Theorem 4. For $\lambda>0, t \in[0, \infty)$ and $x \in \mathbb{R}^{N}$, we define

$$
u_{\lambda}(t, x):=\lambda^{p-q} u\left(\lambda^{2 q-p} t, x_{0}+\lambda^{q-p+1} x\right),
$$

and observe that $u_{\lambda}$ also solves (3) with initial condition $u_{\lambda}(0)$. Furthermore, $u_{\lambda}(0)$ fulfils (9) and, if $\lambda \geq \delta^{-1 /(p-1-q)}$, we infer from (22) that

$$
\begin{aligned}
& \int_{B(0,1)} u_{\lambda}(0, x) d x=\lambda^{p-q+N(p-1-q)} \int_{B\left(x_{0}, \lambda^{q-p+1}\right)} u_{0}(x) d x \\
& \geq A \lambda^{p-q+N(p-1-q)} \int_{B\left(x_{0}, \lambda^{q-p+1}\right)}\left|x-x_{0}\right|^{(p-q) /(p-1-q)} d x \\
& \geq C_{14} A \text {. }
\end{aligned}
$$

Therefore, if $\lambda \geq \delta^{-1 /(p-1-q)}$ and $A \geq A_{q}:=C_{5} / C_{14}$, we infer from Lemma 10 that

$$
\lambda^{p-q} u\left(\lambda^{2 q-p}, x_{0}\right)=u_{\lambda}(1,0) \geq C_{4}\left(\int_{B(0,1)} u_{\lambda}(0, x) d x\right)^{p \eta}>0 .
$$

Consequently, $u\left(t, x_{0}\right)>0$ and thus $x_{0} \in \mathcal{P}(t)$ for $t \in\left(0, \delta^{(p-2 q) /(p-1-q)}\right)$. We finally use the time monotonicity of $\mathcal{P}(t)$ established in Proposition 5 to complete the proof of Theorem 4 .

Combining Theorem 4 with a comparison argument allows us to extend Theorem 4 to $q \in\left[q_{2}, p-1\right)$ under stronger assumptions on the initial data.

Proposition 11 Consider $x_{0} \in \partial \mathcal{P}_{0}$ and assume that there are $A>0, \delta>0$, and $r \in\left(1, q_{2}\right)$ such that

$$
u_{0}(x) \geq A\left|x-x_{0}\right|^{(p-r) /(p-1-r)} \quad \text { for } \quad x \in B\left(x_{0}, \delta\right) .
$$

Then, if $q \in\left[q_{2}, p-1\right)$, we have $x_{0} \in \mathcal{P}(t)$ for all $t>0$. 
Proof. We put $\lambda_{0}:=1 /\left\|\nabla u_{0}\right\|_{\infty}$ and $U(t, x):=\lambda_{0}^{p-q} u\left(\lambda_{0}^{2 q-p} t, \lambda_{0}^{q-p+1} x\right)$ for $(t, x) \in[0, \infty) \times \mathbb{R}^{N}$. Then $U$ solves (3) with initial condition $U_{0}:=U(0)$ and $\left\|\nabla U_{0}\right\|_{\infty}=1$. In addition, $X_{0}:=\lambda_{0}^{p-1-q} x_{0}$ clearly belongs to the boundary of the positivity set of $U_{0}$ and it follows from (39) that

$$
U_{0}(x) \leq \lambda_{0}^{(q-1-r) /(p-1-r)} A\left|x-X_{0}\right|^{(p-r) /(p-1-r)} \quad \text { for } \quad x \in B\left(X_{0}, \delta \lambda_{0}^{p-1-q}\right) .
$$

Introducing $r_{1}:=\left(r+q_{2}\right) / 2 \in\left(r, q_{2}\right)$ we deduce from (40) that, if $\delta_{1} \in\left(0, \delta \lambda_{0}^{p-1-q}\right)$ and $x \in$ $B\left(X_{0}, \delta_{1}\right)$,

$$
\begin{aligned}
U_{0}(x) & \geq \lambda_{0}^{(q-1-r) /(p-1-r)} A\left|x-X_{0}\right|^{\left(p-r_{1}\right) /\left(p-1-r_{1}\right)}\left|x-X_{0}\right|^{\left(r-r_{1}\right) /\left((p-1-r)\left(p-1-r_{1}\right)\right)} \\
& \geq \lambda_{0}^{(q-1-r) /(p-1-r)} \delta_{1}^{\left(r-r_{1}\right) /\left((p-1-r)\left(p-1-r_{1}\right)\right)} A\left|x-X_{0}\right|^{\left(p-r_{1}\right) /\left(p-1-r_{1}\right)},
\end{aligned}
$$

whence

$$
U_{0}(x) \leq A_{1}\left|x-X_{0}\right|^{\left(p-r_{1}\right) /\left(p-1-r_{1}\right)} \quad \text { for } \quad x \in B\left(X_{0}, \delta_{1}\right)
$$

with $A_{1}:=\lambda_{0}^{(q-1-r) /(p-1-r)} \delta_{1}^{\left(r-r_{1}\right) /\left((p-1-r)\left(p-1-r_{1}\right)\right)} A$. Furthermore, we can choose $\delta_{1}$ sufficiently small so that $A \geq A_{r_{1}}$, the constant $A_{r_{1}}$ being defined in Theorem 4 .

Now, on the one hand, it follows from (41) and Theorem 4 that the solution $\tilde{u}$ to

$$
\begin{aligned}
\partial_{t} \tilde{u}-\Delta_{p} \tilde{u}+|\nabla \tilde{u}|^{r_{1}} & =0, \quad(t, x) \in Q, \\
\tilde{u}(0) & =U_{0}, \quad x \in \mathbb{R}^{N},
\end{aligned}
$$

satisfies $\tilde{u}\left(t, X_{0}\right)>0$ for $t>0$. On the other hand, since $\left\|\nabla U_{0}\right\|_{\infty}=1$, we infer from (3) and (12) that

$$
\partial_{t} U-\Delta_{p} U+|\nabla U|^{r_{1}} \geq \partial_{t} U-\Delta_{p} U+\|\nabla U\|_{\infty}^{r_{1}-q}|\nabla U|^{q} \geq \partial_{t} U-\Delta_{p} U+|\nabla U|^{q}=0
$$

in $Q$. The comparison principle [20, Theorem 8.2] then implies that $U(t, x) \geq \tilde{u}(t, x)$ for $(t, x) \in$ $[0, \infty) \times \mathbb{R}^{N}$. Consequently,

$$
u\left(t, x_{0}\right)=\lambda_{0}^{q-p} U\left(\lambda_{0}^{p-2 q} t, X_{0}\right) \geq \lambda_{0}^{q-p} \tilde{u}\left(\lambda_{0}^{p-2 q} t, X_{0}\right)>0
$$

for $t>0$.

\section{Comments, extensions, and open problems}

Theorem 2 gives a precise description of the behaviour for large times of solutions to (3) for $p>2$, $q \in(1, p-1)$, and non-negative and compactly supported initial data. It then gives rise to several related questions, concerning the situation when either the parameters $p$ and $q$ or the initial data do not fulfil the above conditions.

In particular, a natural question is whether a similar result is valid for non-negative initial data which decay rapidly at infinity but are not compactly supported. A first step in that direction would be to identify a class of initial data for which the solutions to (3), (44) satisfy the temporal decay estimates (13). 
Returning to compactly supported initial data, it is tempting to investigate what happens when $q$ reaches the boundary of the range $(1, p-1)$ analyzed here. On the one hand, the critical exponent $q=q_{1}=p-1$ with $p>2$ offers an interesting study of matched asymptotics that we plan to describe in a future publication. On the other hand, the critical case $q=1$ seems to be quite open and interesting, even in the semilinear case [13, 14].

For larger values of $q>p-1$ the situation is expected to be more classical: asymptotic simplification should take place for $q>q_{*}=p-(N /(N+1))$ in the sense that the large time behaviour will be governed by the diffusive part of the equation. For intermediate values of $q \in\left(p-1, q_{*}\right)$, the existence of very singular solutions has been established in [33] and they are expected to describe the large time behaviour of compactly supported solutions.

Concerning waiting times, an interesting technical open problem is to figure out whether Theorem 4 is also true for $q \in\left[q_{2}, q_{1}\right)\left(q_{2}\right.$ is defined in formula (23) $)$.

As a final comment, let us mention that the techniques of this paper could possibly be applied to similar equations involving diffusion and absorption like

$$
\partial_{t} u=\Delta u^{m}-|\nabla u|^{q} \text { in } Q,
$$

but we have noticed that there are a number of difficulties, maybe technical.

Equations with variable coefficients are also worth considering, as well as more general equations of the form (11). In fact, the area of asymptotic behaviour of parabolic equations with variable coefficients is quite open even in the linear case. We refer to [24 for an interesting recent development for linear parabolic equations.

Acknowledgment. This work was started during a visit of $\mathrm{PhL}$ to the Univ. Autónoma de Madrid supported by Spanish Project MTM-2005-08760-C02-01. JLV is partially supported by this project and by ESF Programme "Global and geometric aspects of nonlinear partial differential equations".

\section{References}

[1] Daniele Andreucci, Anatoli F. Tedeev, and Maura Ughi, The Cauchy problem for degenerate parabolic equations with source and damping, Ukrainian Math. Bull. 1 (2004), 1-23.

[2] Stanislav N. Antontsev, Quasilinear parabolic equations with non-isotropic nonlinearities: space and time localization. Energy methods in continuum mechanics (Oviedo, 1994), 1-12, Kluwer Acad. Publ., Dordrecht, 1996.

[3] Stanislav N. Antontsev and Jesús Ildefonso Diaz, On space or time localization of solutions of nonlinear elliptic or parabolic equations via energy methods. Recent advances in nonlinear elliptic and parabolic problems (Nancy, 1988), 3-14, Pitman Res. Notes Math. Ser., 208, Longman Sci. Tech., Harlow, 1989.

[4] Donald G. Aronson, The porous medium equation. Nonlinear diffusion problems (Montecatini Terme, 1985), 1-46, Lecture Notes in Math., 1224, Springer, Berlin, 1986. 
[5] Donald G. Aronson and Luis A. Caffarelli, The initial trace of a solution of the porous medium equation, Trans. Amer. Math. Soc. 280 (1983), 351-366.

[6] Martino Bardi and Italo Capuzzo-Dolcetta, Optimal Control and Viscosity Solutions of Hamilton-Jacobi-Bellman Equations, Systems Control Found. Appl., Birkhäuser, Boston, 1997.

[7] Guy Barles, Asymptotic behavior of viscosity solutions of first order Hamilton-Jacobi equations, Ricerche Mat. 34 (1985), 227-260.

[8] Guy Barles, Solutions de Viscosité des Equations d'Hamilton-Jacobi, Mathématiques \& Applications 17, Springer-Verlag, Berlin, 1994.

[9] Guy Barles and Benoît Perthame, Exit time problems in optimal control and vanishing viscosity method, SIAM J. Control Optim. 26 (1988), 1133-1148.

[10] Jean-Philippe Bartier and Philippe Laurençot, Gradient estimates for a degenerate parabolic equation with gradient absorption and applications, (submitted).

[11] Saïd Benachour, Grzegorz Karch, and Philippe Laurençot, Asymptotic profiles of solutions to viscous Hamilton-Jacobi equations, J. Math. Pures Appl. (9) 83 (2004), 1275-1308.

[12] Saïd Benachour, Philippe Laurençot, Didier Schmitt, and Philippe Souplet, Extinction and non-extinction for viscous Hamilton-Jacobi equations in $\mathbb{R}^{N}$, Asymptot. Anal. 31 (2002), 229246.

[13] Saïd Benachour, Bernard Roynette, and Pierre Vallois, Solutions fondamentales de $u_{t}-\frac{1}{2} u_{x x}=$ $\pm\left|u_{x}\right|$, Astérisque 236 (1996), 41-71.

[14] Saïd Benachour, Bernard Roynette, and Pierre Vallois, Asymptotic estimates of solutions of $u_{t}-\frac{1}{2} \Delta u=-|\nabla u|$ in $\mathbb{R}_{+} \times \mathbb{R}^{d}, d \geq 2$, J. Funct. Anal. 144 (1997), 301-324.

[15] Michiel Bertsch, Tokumori Nanbu, and Lambertus A. Peletier, Decay of solutions of a degenerate nonlinear diffusion equation, Nonlinear Anal. 6 (1982), 539-554.

[16] Michiel Bertsch, Robert Kersner, and Lambertus A. Peletier, Sur le comportement de la frontière libre dans une équation en théorie de la filtration, C. R. Acad. Sci. Paris Sér. I 295 (1982), 63-66.

[17] Piotr Biler, Mohammed Guedda, and Grzegorz Karch, Asymptotic properties of solutions of the viscous Hamilton-Jacobi equation, J. Evolution Equations 4 (2004), 75-97.

[18] Manuela Chaves and Juan Luis Vázquez, Nonuniqueness in nonlinear heat propagation: a heat wave coming from infinity, Differential Integral Equations 9 (1996), 447-464.

[19] Manuela Chaves and Juan Luis Vázquez, Free boundary layer formation in nonlinear heat propagation, Comm. Partial Differential Equations 24 (1999), 1945-1965.

[20] Michael G. Crandall, Hitoshi Ishii, and Pierre-Louis Lions, User's guide to viscosity solutions of second order partial differential equations, Bull. Amer. Math. Soc. (N.S.) 27 (1992), 1-67. 
[21] Jesús Ildefonso Diaz and Laurent Véron, Local vanishing properties of solutions of elliptic and parabolic quasilinear equations, Trans. Amer. Math. Soc. 290 (1985), 787-814.

[22] Thierry Gallay and Philippe Laurençot, Asymptotic behavior for a viscous Hamilton-Jacobi equation with critical exponent, Indiana Univ. Math. J. 56 (2007), 459-479.

[23] Brian H. Gilding, The Cauchy problem for $u_{t}=\Delta u+|\nabla u|^{q}$, large-time behaviour, J. Math. Pures Appl. (9) 84 (2005), 753-785.

[24] Juraj Húska, Peter Poláčik, and Mikhail V. Safonov, Harnack inequalities, exponential separation, and perturbations of principal Floquet bundles for linear parabolic equations, Preprint, 2007.

[25] Abdelilah Gmira and Benyouness Bettioui, On the selfsimilar solutions of a diffusion convection equation, NoDEA Nonlinear Differential Equations Appl. 9 (2002), 277-294.

[26] Hitoshi Ishii, A simple, direct proof of uniqueness for solutions of the Hamilton-Jacobi equations of eikonal type, Proc. Amer. Math. Soc. 100 (1987), 247-251.

[27] Anatolii S. Kalashnikov, Some problems of the qualitative theory of non-linear degenerate second-order parabolic equations, Russian Math. Surveys 42 (1987), 169-222.

[28] Shoshana Kamin and Juan Luis Vázquez, Fundamental solutions and asymptotic behaviour for the p-Laplacian equation, Rev. Mat. Iberoamericana 4 (1988), 339-354.

[29] Barry F. Knerr The porous medium equation in one dimension, Trans. Amer. Math. Soc. 234 (1977), 381-415.

[30] Olga A. Ladyzhenskaya, Vsevolod A. Solonnikov, and Nina N. Uraltseva. Linear and Quasilinear Equations of Parabolic Type, Amer. Math. Soc., Providence, R.I. 1968.

[31] Gawtum Namah and Jean-Michel Roquejoffre, Remarks on the long time behaviour of the solutions of Hamilton-Jacobi equations, Comm. Partial Differential Equations 24 (1999), 883893.

[32] Jean-Michel Roquejoffre, Convergence to steady states or periodic solutions in a class of Hamilton-Jacobi equations, J. Math. Pures Appl. (9) 80 (2001), 85-104.

[33] Shi Peihu, Self-similar singular solution of a p-Laplacian evolution equation with gradient absorption term, J. Partial Differential Equations 17 (2004), 369-383.

[34] Juan Luis Vázquez, Singular solutions and asymptotic behaviour of nonlinear parabolic equations, International Conference on Differential Equations, Vol. 1, 2 (Barcelona, 1991), 234-249, World Sci. Publ., River Edge, NJ, 1993.

[35] Juan Luis Vázquez, Smoothing and decay estimates for nonlinear diffusion equations. Equations of porous medium type, Oxford Lecture Ser. Math. Appl. 33, Oxford University Press, Oxford, 2006.

[36] Juan Luis Vázquez, The porous medium equation. Mathematical theory, Oxford Mathematical Monographs. The Clarendon Press, Oxford University Press, Oxford, 2007. 
[37] Yuan Hongjun, Localization condition for a nonlinear diffusion equation, Chinese J. Contemp. Math. 17 (1996), 45-58.

Keywords: nonlinear parabolic equations, $p$-Laplacian equation, asymptotic patterns, localization, Hamilton-Jacobi equations, viscosity solutions. 